\title{
Interaction Between an Obliquely Incident Plane Electromagnetic Wave and an Electron Beam in the Presence of a Static Magnetic Field of Arbitrary Strength
}

\author{
K. H. B. Wilhelmsson \\ Contribution from Research Laboratory of Electronics, Chalmers University of Technology, \\ Gothenburg, Sweden \\ (Received December 11, 1961)

\begin{abstract}
The purpose of the paper is to study theoretically the interaction between an obliquely incident plane electromagnetic wave and an electron beam. We assume that a static magnetic field of arbitrary strength is present in the axial direction.

Machine computations made for the case of a cylindrical electron plasma show that resonances occur in the backscattering cross section as a function of the angle of incidence of the plane wave. The dependence of the resonance angles on the plasma frequency for fixed gyrofrequency suggests a possibility of utilizing the results of the investigation for diagnostics of a cylindrical plasma.
\end{abstract}

\section{Introduction and Summary}

The interaction of an obliquely incident plane electromagnetic wave and an electron beam has been studied by the author in a number of previous reports [Wilhelmsson, 1954, 1958a, 1958b], particularly in the presence of an infinitely strong static magnetic field in the direction of the beam and in the absence of a static magnetic field. In an earlier report [Wilhelmsson, $1958 \mathrm{c}]$ the author discussed the wave solutions for the waves excitable in a cylindrical electron beam in the presence of a static magnetic field of arbitrary strength. The investigation also covered the case of azimuthally asymmetric modes which, together with the azimuthally symmetric modes, constitute the complete set of normal modes excitable, e.g., by means of an obliquely incident plane wave.

The purpose of the present report is to give the complete formal solution of the problem of interaction between an obliquely incident plane wave and an electron beam in the presence of a static magnetic field of finite strength in the axial direction.

The investigation suggests a new interesting diagnostic technique for a beam or a plasma utilizing the dependence of the resonance angles of incidence on the plasma and gyrofrequencies.

\section{General Assumptions and Fundamental Equations}

Let us review the assumptions and the notations introduced by the author in previous communications on this subject. We consider a circular electron beam of radius $a$ and of infinite extent in the axial direction. The beam has a drift velocity $v_{0}$ along the lines of force of a homogeneous magnetic field of strength $H_{0}$. We assume that the beam travels through a neutral ionized medium that exists only in the same domain as the beam. The static electron densities of the beam and the ionized medium are further assumed to be homogeneous in space. Let us introduce the critical angular frequencies $\omega_{c 1}=\left(N_{1} e^{2} / m \epsilon_{0}\right)^{1 / 2}$ and $\omega_{c 2}=\left(N_{2} e^{2} / m \epsilon_{0}\right)^{1 / 2}$ corresponding to the electron densities $N_{1}$ and $N_{2}$ of the beam and the ionized medium, respectively. Here $e$ is the charge of the electron, $m$ its mass, and $\epsilon_{0}$ the dielectric constant of vacuum. In the following we consider frequencies of an order of magnitude such that the ions can be regarded at rest. 


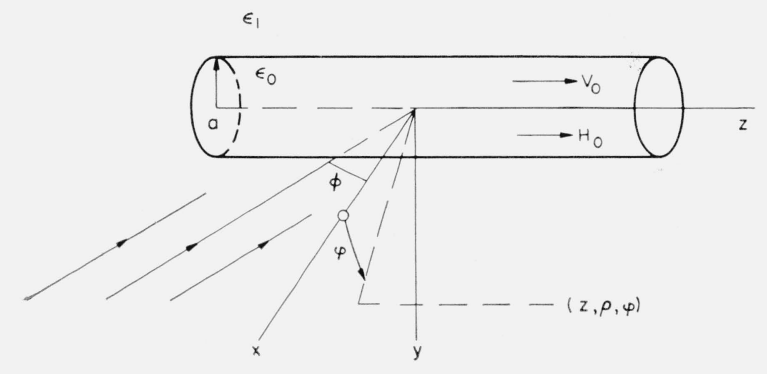

FiguRE 1. The obliquely incident plane electromagnetic wave and the electron beam.

In order to study the interaction between an obliquely incident plane electromagnetic wave and an electron beam in the presence of a static magnetic field of finite amplitude, we introduce circular cylindrical coordinates $(z, \rho, \varphi)$ and assume that the incident plane wave hits the beam with an angle of incidence $\phi$. We further assume that the dielectric constants are different outside and inside the beam. By virtue of the last assumption, the axial phase velocity of the incident wave can be adjusted to the velocity of the beam by a suitable choice of the dielectric constant of the surrounding medium.

We represent the axial wave propagation by the factor $e^{j\left(\omega t-k_{\|} z\right)}$, where $\omega$ is the angular frequency of the oscillations, $t$ the time, $k_{\|}=k_{1} \sin \phi$, where $k_{1}=\omega \sqrt{\mu_{1} \epsilon_{1}}$ is the wave propagation constant of the incident plane wave $\left(\mu_{1}\right.$ and $\epsilon_{1}$ are the permeability and the dielectric constant of the surrounding medium). A convenient notation is further $k_{\perp}=k_{1} \cos \phi . \quad z$ is the axial space coordinate. For vacuum we introduce correspondingly $k_{0}=\omega \sqrt{\mu_{0} \epsilon_{0}}$. The wave impedances we denote by $Z_{1}=\sqrt{\frac{\mu_{1}}{\epsilon_{1}}}$ and $Z_{0}=\sqrt{\frac{\mu_{0}}{\epsilon_{0}}}$. We represent the angular dependence of the wave functions by the factor $e^{j n \varphi}$.

The cyclotron frequency of the electrons we denote by $\omega_{H}=\frac{e}{m} \mu_{0} H_{0}$, where $\mu_{0}$ is the vacuum permeability. We furthermore make use of the notation $\alpha_{1}=\frac{\omega}{v_{0}}$, "the phase constant of the electron beam," and $k_{p_{0}}=\frac{2 \pi}{\lambda_{p_{0}}}=\frac{\omega_{c_{1}}}{v_{0}}$, the relative space charge wave propagation constant, where $\lambda_{p_{0}}$ is the plasmic wavelength of an infinitely wide beam. Collisional effects are taken into account by introducing the effective collision frequencies $\nu_{1}$ and $\nu_{2}$ for the electrons of the beam and for the electrons of the ionized medium respectively.

In a previous investigation [Wilhelmsson, 1958c] the author obtained the following coupled equations for the quantities $\Pi_{1}$ and $\Pi_{2}$ defined by $\Pi_{1}=E_{z}$ and $\Pi_{2}=\sqrt{\frac{\mu_{0}}{\epsilon_{0}}} H_{z}$

$$
\begin{aligned}
& \left(P_{\rho}+A_{1}^{2}\right) \Pi_{1}=B_{1}^{2} \Pi_{2}, \\
& \left(P_{\rho}+A_{2}^{2}\right) \Pi_{2}=B_{2}^{2} \Pi_{1},
\end{aligned}
$$

where $P_{\rho}$ is the operator

$$
P_{\rho}=\frac{1}{\rho} \frac{\partial}{\partial \rho} \rho \frac{\partial}{\partial \rho}-\frac{n^{2}}{\rho^{2}}
$$

and $A_{1}, A_{2}, B_{1}$, and $B_{2}$ depend on the parameters we have already defined.

An alternative way of writing the eqs (1) and (2) is

$$
\begin{aligned}
& \left(P_{\rho}+a_{1}^{2}\right) \Pi_{1}=b_{1}^{2} P_{\rho} \Pi_{2}, \\
& \left(P_{\rho}+a_{2}^{2}\right) \Pi_{2}=b_{2}^{2} P_{\rho} \Pi_{1},
\end{aligned}
$$

where again $a_{1}, a_{2}, b_{1}$, and $b_{2}$ can be expressed in the parameters that we have introduced.

The equations were derived from the equation of motion of the electrons, the equation of continuity, and Maxwell's equations, taking into consideration the drift Lorentz term $\bar{v}_{0} \times \bar{H}_{\sim}$, where $\bar{H}_{\sim}$ is the a-c magnetic field, and the relativistic effects. Let us here introduce the 
notation $\beta=\frac{v_{0}}{c_{0}}$ for the ratio between the drift velocity of the electron beam and the velocity of light in vacuum.

We obtain the following expressions for the constants in the coupled field equations

$$
\begin{gathered}
a_{1}^{2}=\frac{\Delta^{2}}{p^{2}}\left\{k_{0}^{2}\left[1-\frac{\omega_{c_{1}}^{2}}{\omega^{2}} \cdot \frac{\omega-v_{0} k_{\|}}{\omega-v_{0} k_{\|}-\omega_{H}\left(1-\beta^{2}\right)^{\frac{1}{2}}-j \nu_{1}}\left(1-\beta^{2}\right)^{\frac{1}{2}}-\frac{\omega_{c_{2}}^{2}}{\omega\left(\omega-\omega_{H}-j \nu_{2}\right)}\right]-k_{\|}^{2}\right\} \\
\times\left\{k_{0}^{2}\left[1-\frac{\omega_{c_{1}}^{2}}{\omega^{2}} \cdot \frac{\omega-v_{0} k_{\|}}{\omega-v_{0} k_{\|}+\omega_{H}\left(1-\beta^{2}\right)^{\frac{1}{2}}-j \nu_{1}}\left(1-\beta^{2}\right)^{\frac{1}{2}}-\frac{\omega_{c_{2}}^{2}}{\omega\left(\omega+\omega_{H}-j \nu_{2}\right)}\right]-k_{\|}^{2}\right\}, \\
a_{2}^{2}=\frac{1}{q^{2}}\left\{k_{0}^{2}\left[1-\frac{\omega_{c_{1}}^{2}}{\omega^{2}} \cdot \frac{\omega-v_{0} k_{\|}}{\omega-v_{0} k_{\|}-\omega_{H}\left(1-\beta^{2}\right)^{\frac{1}{2}}-j \nu_{1}}\left(1-\beta^{2}\right)^{\frac{1}{2}}-\frac{\omega_{c_{2}}^{2}}{\omega\left(\omega-\omega_{H}-j \nu_{2}\right)}\right]-k_{\|}^{2}\right\} \\
\times\left\{k_{0}^{2}\left[1-\frac{\omega_{c_{1}}^{2}}{\omega^{2}} \cdot \frac{\omega-v_{0} k_{\|}}{\omega-v_{0} k_{\|}+\omega_{H}\left(1-\beta^{2}\right)^{\frac{1}{2}}-j \nu_{1}}\left(1-\beta^{2}\right)^{\frac{1}{2}}-\frac{\omega_{c_{2}}^{2}}{\omega\left(\omega+\omega_{H}-j \nu_{2}\right)}\right]-k_{\|}^{2}\right\}, \\
b_{1}^{2}=j \frac{\Lambda}{p^{2}}, \quad b_{2}^{2}=-j \frac{\Lambda}{q^{2}}, \\
A_{1}^{2}=q^{2} \cdot \frac{\Delta^{2}}{D^{2}}, \quad A_{2}^{2}=q^{2}-k_{0}^{2} \frac{T^{2}}{D^{2}}, \\
B_{1}^{2}=-j \frac{\Lambda}{D^{2}}, \quad B_{2}^{2}=j \Lambda \frac{\Delta^{2}}{D^{2}},
\end{gathered}
$$

where

$$
\begin{aligned}
& q^{2}=k_{0}^{2}\left[1-\frac{\omega_{c_{1}}^{2}}{\left(\omega-v_{0} k_{\|}-j \nu_{1}\right)^{2}-\omega_{H}^{2}\left(1-\beta^{2}\right)} \cdot \frac{\left(\omega-v_{0} k_{\|}-j \nu_{1}\right)\left(\omega-v_{0} k_{\|}\right)}{\omega^{2}}\left(1-\beta^{2}\right)^{\frac{1}{2}}-\frac{\omega_{c_{2}}^{2}}{\left(\omega-j \nu_{2}\right)^{2}-\omega_{H}^{2}} \cdot \frac{\omega-j \nu_{2}}{\omega}\right]-k_{\|}^{2}, \\
& \Delta^{2}=1-\frac{\omega_{c_{1}}^{2}}{\left(\omega-v_{0} k_{\|}\right)\left(\omega-v_{0} k_{\|}-j \nu_{1}\right)}\left(1-\beta^{2}\right)^{\frac{3}{2}}-\frac{\omega_{c_{2}}^{2}}{\omega\left(\omega-j \nu_{2}\right)}, \\
& D^{2}=1-\frac{\omega_{c_{1}}^{2}}{\left(\omega-v_{0} k_{\|}-j \nu_{1}\right)^{2}-\omega_{H}^{2}\left(1-\beta^{2}\right)} \cdot \frac{\omega-v_{0} k_{\|}-j \nu_{1}}{\omega-v_{0} k_{\|}}\left(1-\beta^{2}\right)^{\frac{3}{2}}-\frac{\omega_{c_{2}}^{2}}{\left(\omega-j \nu_{2}\right)^{2}-\omega_{H}^{2}} \cdot \frac{\omega-j \nu_{2}}{\omega} \\
& \times\left[1+\beta^{2} \cdot \frac{\omega_{c_{1}}^{2}}{\left(\omega-v_{0} k_{\|}-j \nu_{1}\right)^{2}-\omega_{H}^{2}\left(1-\beta^{2}\right)} \cdot \frac{\omega-v_{0} k_{\|}-j \nu_{1}}{\omega-v_{0} k_{\|}}\left(1-\beta^{2}\right)^{\frac{1}{2}}\right], \\
& T^{2}=\frac{\omega_{H}^{2}}{\omega^{2}}\left(\left[\frac{\omega_{c_{1}}^{2}}{\left(\omega-v_{0} k_{\|}-j \nu_{1}\right)^{2}-\omega_{H}^{2}\left(1-\beta^{2}\right)}\left(1-\beta^{2}\right)+\frac{\omega_{c_{2}}^{2}}{\left(\omega-j \nu_{2}\right)^{2}-\omega_{H}^{2}}\right]^{2}\right. \\
& -\beta^{2} \frac{\omega_{c_{1}}^{2}}{\left(\omega-v_{0} k_{\|}-j \nu_{1}\right)^{2}-\omega_{H}^{2}\left(1-\beta^{2}\right)}\left(1-\beta^{2}\right)^{\frac{1}{2}} \cdot\left\{\frac{\omega_{c_{1}}^{2}}{\left(\omega-v_{0} k_{\|}-j \nu_{1}\right)^{2}-\omega_{H}^{2}\left(1-\beta^{2}\right)}\left(1-\beta^{2}\right)^{\frac{3}{2}}\right. \\
& \left.\left.-\frac{\omega_{c_{2}}^{2}}{\left(\omega-j \nu_{2}\right)^{2}-\omega_{H}^{2}}\left[\frac{\omega_{c_{1}}^{2}}{\left(\omega-v_{0} k_{\|}-j \nu_{1}\right)^{2}-\omega_{H}^{2}\left(1-\beta^{2}\right)} \cdot \frac{\omega-j \nu_{2}}{\omega}\left(1-\beta^{2}\right)^{\frac{3}{2}}+\frac{\omega_{c_{2}}^{2}}{\left(\omega-j \nu_{2}\right)^{2}-\omega_{H}^{2}} \cdot \frac{\omega-v_{0} k_{\|}-j \nu_{1}}{\omega-v_{0} k_{\|}}\right]\right\}\right), \\
& p^{2}=q^{2} D^{2}-k_{0}^{2} T^{2}, \\
& \Lambda=-k_{0} \frac{\omega_{H}}{\omega}\left(\frac{k_{0}^{2}-\alpha_{1} k_{\|}}{\alpha_{1}} \cdot \frac{\omega_{c_{1}}^{2}}{\left(\omega-v_{0} k_{\|}-j \nu_{1}\right)^{2}-\omega_{H}^{2}\left(1-\beta^{2}\right)}\left(1-\beta^{2}\right)\right. \\
& -\frac{\omega_{c_{2}}^{2}}{\left(\omega-j \nu_{2}\right)^{2}-\omega_{H}^{2}}\left\{k_{\|}+\beta^{2} \frac{\omega_{c_{1}}^{2}}{\left(\omega-v_{0} k_{\|}-j \nu_{1}\right)^{2}-\omega_{H}^{2}\left(1-\beta^{2}\right)}\left(1-\beta^{2}\right)^{\frac{1}{2}}\right. \\
& \left.\left.\times\left[k_{\|}-\alpha_{1}+j \frac{\nu_{1}}{v_{0}}+\left(\alpha_{1}-j \frac{\nu_{2}}{v_{0}}\right)\left(1-\beta^{2}\right)^{\frac{1}{2}}\right]\right\}\right) .
\end{aligned}
$$


The quantities that we have introduced are sufficient to determine the coupled eqs $(1,2)$ and (1a, 2a) completely. To construct expressions for all the field components that we need to satisfy the boundary conditions on the surface of the cylinder, it is convenient to introduce the following quantities:

$$
\begin{aligned}
& Q=\frac{\omega_{H}}{\omega}\left[\frac{\omega_{c_{1}}^{2}}{\left(\omega-v_{0} k_{\|}-j \nu_{1}\right)^{2}-\omega_{H}^{2}\left(1-\beta^{2}\right)} \cdot \frac{\omega-v_{0} k_{\|}}{\omega}\left(1-\beta^{2}\right)+\frac{\omega_{c_{2}}^{2}}{\left(\omega-j \nu_{2}\right)^{2}-\omega_{H}^{2}}\right], \\
& R=\frac{q^{2}}{k_{0}^{2}}\left[1-\frac{\omega_{c_{1}}^{2}}{\left(\omega-v_{0} k_{\|}-j \nu_{1}\right)^{2}-\omega_{H}^{2}\left(1-\beta^{2}\right)} \cdot \frac{\omega-v_{0} k_{\|}-j \nu_{1}}{\omega}\left(1-\beta^{2}\right)^{\frac{1}{2}}-\frac{\omega_{c_{2}}^{2}}{\left(\omega-j \nu_{2}\right)^{2}-\omega_{H}^{2}} \cdot \frac{\omega-j \nu_{2}}{\omega}\right] \\
& \quad-\frac{\omega_{H}}{\omega} Q\left[\frac{\omega_{c_{1}}^{2}}{\left(\omega-v_{0} k_{\|}-j \nu_{1}\right)^{2}-\omega_{H}^{2}\left(1-\beta^{2}\right)}\left(1-\beta^{2}\right)+\frac{\omega_{c_{2}}^{2}}{\left(\omega-j \nu_{2}\right)^{2}-\omega_{H}^{2}}\right], \\
& \Gamma=k_{\|}\left[1-\frac{k_{0}^{2}}{\alpha_{1} k_{\|}} \cdot \frac{\omega_{c_{1}}^{2}}{\left(\omega-v_{0} k_{\|}-j \nu_{1}\right)^{2}-\omega_{H}^{2}\left(1-\beta^{2}\right)} \cdot \frac{\omega-v_{0} k_{\|}-j \nu_{1}}{\omega}\left(1-\beta^{2}\right)^{\frac{1}{2}}\right] q^{2} \\
& -\frac{k_{0}^{4}}{\alpha_{1}} \cdot \frac{\omega_{c_{1}}^{2}}{\left(\omega-v_{0} k_{\|}-j \nu_{1}\right)^{2}-\omega_{H}^{2}\left(1-\beta^{2}\right)}\left(1-\beta^{2}\right) \frac{\omega_{H}}{\omega} Q . \quad(3 \mathrm{~b})
\end{aligned}
$$

\section{Wave Solutions of the Problem}

We consider two different directions of polarization of the obliquely incident plane electromagnetic wave, viz, the cases with the magnetic or the electric field vector polarized perpendicularly to the electron beam. The general solution for the case of an arbitrarily polarized incident plane wave can be obtained by superposition of the solutions for the two separate cases.

\subsection{The Magnetic Field Vector of the Incident Plane Wave Is Perpendicular to the Electron Beam}

The field vectors of the incident plane electromagnetic wave can be written

$$
\begin{aligned}
& E^{\mathrm{inc}}=A e^{j\left(\omega t+k_{\perp \rho} \cos \varphi-k_{\|} z\right)}, \\
& H^{\mathrm{inc}}=A \frac{1}{Z_{1}} e^{j\left(\omega t+k \perp \rho \cos \varphi-k_{\|} z\right)} .
\end{aligned}
$$

From the Jacobi series

$$
e^{j k \perp \rho \cos \varphi}=\sum_{n=-\infty}^{\infty} j^{n} J_{n}\left(k_{\perp} \rho\right) e^{j n \varphi}
$$

we deduce the following expansions that we need to express the field components of the incident plane wave in a suitable form

$$
\begin{aligned}
\sin \varphi e^{j k_{\perp \rho} \cos \varphi} & =\frac{-1}{j k_{\perp} \rho} \frac{d}{d \varphi} e^{j k_{\perp \rho} \cos \varphi}=\frac{-1}{k_{\perp} \rho} \sum_{n=-\infty}^{\infty} n j^{n} J_{n}\left(k_{\perp} \rho\right) e^{j n \varphi}, \\
\cos \varphi e^{j k_{\perp \rho} \cos \varphi} & =-j \frac{d}{d\left(k_{\perp} \rho\right)} e^{j k_{\perp \rho} \cos \varphi}=\sum_{n=-\infty}^{\infty} j^{n-1} J_{n}^{\prime}\left(k_{\perp} \rho\right) e^{j n \varphi} .
\end{aligned}
$$

We then obtain (the axial propagation factor $e^{j\left(\omega t-k_{\|} z\right)}$ is omitted in the following)

$$
\begin{aligned}
& E_{z}^{\mathrm{inc}}=-E^{\mathrm{inc}} \cos \phi=-A \cos \phi \sum_{n=-\infty}^{\infty} j^{n} J_{n}\left(k_{\perp} \rho\right) e^{j n \varphi} \\
& E_{\varphi}^{\mathrm{inc}}=E^{\mathrm{inc}} \sin \phi \sin \varphi=-A \sin \phi \frac{1}{k_{\perp} \rho} \sum_{n=-\infty}^{\infty} n j^{n} J_{n}\left(k_{\perp} \rho\right) e^{j n \varphi} \\
& H_{\varphi}^{\mathrm{inc}}=-H^{\mathrm{inc}} \cos \varphi=-A \frac{1}{Z_{1}} \sum_{n=-\infty}^{\infty} j^{n-1} J_{n}^{\prime}\left(k_{\perp} \rho\right) e^{j n \varphi} .
\end{aligned}
$$


The general solution of the coupled field equations (1) and (2) that we need in order to describe the internal field, we write in the following form

$$
\begin{aligned}
& E_{z}^{\mathrm{int}}=\sum_{n=-\infty}^{\infty} A_{1 n} J_{n}\left(h_{1} \rho\right) e^{j n \varphi}+\frac{B_{1}^{2}}{A_{2}^{2}-h_{2}^{2}} Z_{0} \sum_{n=-\infty}^{\infty} B_{1 n} J_{n}\left(h_{2} \rho\right) e^{j n \varphi}, \\
& H_{z}^{\mathrm{int}}=\frac{B_{2}^{2}}{A_{2}^{2}-h_{1}^{2}} \frac{1}{Z_{0}} \sum_{n=-\infty}^{\infty} A_{1 n} J_{n}\left(h_{1} \rho\right) e^{j n \varphi}+\sum_{n=-\infty}^{\infty} B_{1 n} J_{n}\left(h_{2} \rho\right) e^{j n \varphi}
\end{aligned}
$$

where $h_{1}$ and $h_{2}$ satisfy the relation

$$
\left(h^{2}-A_{1}^{2}\right)\left(h^{2}-A_{2}^{2}\right)=B_{1}^{2} B_{2}^{2}
$$

In eqs (9) and (10) $A_{1 n}$ and $B_{1 n}$ are amplitude coefficients, which are to be evaluated later on by introducing boundary conditions. Since $J_{n}(-z)=(-1)^{n} \boldsymbol{J}_{n}(z)$ it is sufficient in eqs (9) and (10) to consider only two roots $h_{1}$ and $h_{2}$ of the 4th-order equation (11) that do not differ only by sign in order to construct the complete solution for the internal field.

The second term of the right-hand side of eq (9) and the first term of the right-hand side of eq (10) are terms introduced by the coupling expressed by the right-hand sides of eqs (1) and (2). The remaining terms of eqs (9) and (10) correspond in the limit of infinitely strong static magnetic field to pure transverse magnetic (TM) and transverse electric (TE) modes respectively.

We choose

so that

$$
\begin{aligned}
& h_{1}^{2}=\frac{1}{2}\left[A_{1}^{2}+A_{2}^{2}-\sqrt{\left.\left(A_{2}^{2}-A_{1}^{2}\right)^{2}+4 B_{1}^{2} B_{2}^{2}\right]},\right. \\
& h_{2}^{2}=\frac{1}{2}\left[A_{1}^{2}+A_{2}^{2}+\sqrt{\left.\left(A_{2}^{2}-A_{1}^{2}\right)^{2}+4 B_{1}^{2} B_{2}^{2}\right]},\right.
\end{aligned}
$$

and

$$
\lim _{\omega_{H \rightarrow \infty}} h_{1}=A_{1}
$$

$$
\lim _{\omega_{H} \rightarrow \infty} h_{2}=A_{2} .
$$

We furthermore have the relations

$$
\begin{aligned}
A_{2}^{2}-A_{1}^{2} & =\frac{1}{D^{2}}\left(p^{2}-q^{2} \Delta^{2}\right), \\
B_{1}^{2} B_{2}^{2} & =\Lambda^{2} \frac{\Delta^{2}}{D^{4}} .
\end{aligned}
$$

Hence

$$
\begin{aligned}
& A_{1}^{2}-h_{2}^{2}=-\frac{1}{2}\left[A_{2}^{2}-A_{1}^{2}+\sqrt{\left.\left(A_{2}^{2}-A_{1}^{2}\right)^{2}+4 B_{1}^{2} B_{2}^{2}\right]}=-\frac{1}{2 D^{2}}\left[p^{2}-q^{2} \Delta^{2}+\sqrt{\left.\left(p^{2}-q^{2} \Delta^{2}\right)^{2}+4 \Lambda^{2} \Delta^{2}\right]},\right.\right. \\
& A_{2}^{2}-h_{1}^{2}=\frac{1}{2}\left[A_{2}^{2}-A_{1}^{2}+\sqrt{\left.\left(A_{2}^{2}-A_{1}^{2}\right)^{2}+4 B_{1}^{2} B_{2}^{2}\right]}=\frac{1}{2 D^{2}}\left[p^{2}-q^{2} \Delta^{2}+\sqrt{\left.\left(p^{2}-q^{2} \Delta^{2}\right)^{2}+4 \Lambda^{2} \Delta^{2}\right]},\right.\right.
\end{aligned}
$$

and

$$
\begin{aligned}
& h_{1}^{2}=\frac{1}{2 D^{2}}\left[p^{2}+q^{2} \Delta^{2}-\sqrt{\left.\left(p^{2}-q^{2} \Delta^{2}\right)^{2}+4 \Lambda^{2} \Delta^{2}\right]},\right. \\
& h_{2}^{2}=\frac{1}{2 D^{2}}\left[p^{2}+q^{2} \Delta^{2}+\sqrt{\left.\left(p^{2}-q^{2} \Delta^{2}\right)^{2}+4 \Lambda^{2} \Delta^{2}\right]} .\right.
\end{aligned}
$$

Let us introduce

$$
U=\frac{1}{2 \Lambda}\left[q^{2} \Delta^{2}-p^{2}+\sqrt{\left.\left(p^{2}-q^{2} \Delta^{2}\right)^{2}+4 \Lambda^{2} \Delta^{2}\right]} .\right.
$$


We then have

$$
\begin{aligned}
& \frac{B_{1}^{2}}{A_{1}^{2}-h_{2}^{2}}=j U \Delta^{-2}, \\
& \frac{B_{2}^{2}}{A_{2}^{2}-h_{1}^{2}}=j U .
\end{aligned}
$$

The internal wave solutions (9) and (10) can therefore be written

$$
\begin{aligned}
& E_{z}^{\text {int }}=\sum_{n=-\infty}^{\infty} A_{1 n} J_{n}\left(h_{1} \rho\right) e^{j n \varphi}+j U \Delta^{-2} Z_{0} \sum_{n=-\infty}^{\infty} B_{1 n} J_{n}\left(h_{2} \rho\right) e^{j n \varphi}, \\
& H_{z}^{\text {int }}=j U \frac{1}{Z_{0}} \sum_{n=-\infty}^{\infty} A_{1 n} J_{n}\left(h_{1} \rho\right) e^{j n \varphi}+\sum_{n=-\infty}^{\infty} B_{1 n} J_{n}\left(h_{2} \rho\right) e^{j n \varphi} .
\end{aligned}
$$

For the azimuthal components of the internal electric and magnetic fields we have the following expressions in $E_{z}^{\text {int }}, H_{z}^{\text {int }}$ and their first radial derivatives

$$
\begin{aligned}
& E_{\varphi}^{\mathrm{int}}=\frac{1}{q^{2} a_{2}^{2}}\left[\Gamma \frac{n}{\rho} E_{z}^{\mathrm{int}}-k_{0} \Lambda \frac{\partial E_{z}^{\mathrm{int}}}{\partial \rho}-j k_{0}^{3} Z_{0} Q \frac{n}{\rho} H_{z}^{\mathrm{int}}+j k_{0} Z_{0} q^{2} \frac{\partial H_{z}^{\mathrm{int}}}{\partial \rho}\right] \\
& H_{\varphi}^{\mathrm{int}}=\frac{1}{q^{2} a_{2}^{2}}\left[j \frac{k_{\|}}{Z_{0}} \Lambda \frac{n}{\rho} E_{z}^{\mathrm{int}}-j \frac{k_{0}^{3}}{Z_{0}} R \frac{\partial E_{z}^{\mathrm{int}}}{\partial \rho}+k_{\|} q^{2} \frac{n}{\rho} H_{z}^{\mathrm{int}}-k_{0}^{2} k_{\|} Q \frac{\partial H_{z}^{\mathrm{int}}}{\partial \rho}\right] .
\end{aligned}
$$

The formulae (17) and (18) contain sufficient information on the internal electromagnetic field to determine the amplitude coefficients by introducing the boundary conditions on the surface of the cylinder. The determination of the amplitude coefficients will be the subject of a following section of the investigation.

The radial components of the internal fields may, if desired, be obtained from the following expressions and relations (17) and (18)

$$
\begin{aligned}
& E_{\rho}^{\mathrm{int}}=\frac{1}{k_{\|}}\left(k_{0} Z_{0} H_{\varphi}^{\mathrm{int}}+j \frac{\partial E_{z}^{\mathrm{int}}}{\partial \rho}\right), \\
& H_{\rho}^{\mathrm{int}}=-\frac{1}{k_{0} Z_{0}}\left(k_{\mathbb{}} E_{\varphi}^{\mathrm{int}}+\frac{n}{\rho} E_{z}^{\mathrm{int}}\right) .
\end{aligned}
$$

We write the field components of the scattered wave $(\rho>a)$ in the following way

TM modes

$$
\begin{aligned}
& E_{z}^{s c}=\sum_{n=-\infty}^{\infty} A_{2 n} H_{n}^{(2)}\left(k_{\perp} \rho\right) e^{j n \varphi} \\
& E_{\rho}^{s c}=-j \tan \phi \sum_{n=-\infty}^{\infty} A_{2 n} H_{n}^{(2)^{\prime}}\left(k_{\perp} \rho\right) e^{j n \varphi} \\
& E_{\varphi}^{s c}=\frac{\tan \phi}{k_{\perp} \rho} \sum_{n=-\infty}^{\infty} n A_{2 n} H_{n}^{(2)}\left(k_{\perp} \rho\right) e^{j n \varphi} \\
& H_{\rho}^{s c}=-\frac{1}{Z_{1}} \cdot \frac{1}{k_{\perp} \rho \cos \phi} \sum_{n=-\infty}^{\infty} n A_{2 n} H_{n}^{(2)}\left(k_{\perp} \rho\right) e^{j n \varphi} \\
& H_{\rho}^{s c}=-j \frac{1}{Z_{1}} \cdot \frac{1}{\cos \phi} \sum_{n=-\infty}^{\infty} A_{2 n} H_{n}^{(2)^{\prime}}\left(k_{\perp} \rho\right) e^{j n \varphi} \\
& H_{z}^{s c}=0
\end{aligned}
$$




$$
\begin{aligned}
& E_{z}^{s c}=0 \\
& E_{\rho}^{s c}=Z_{1} \frac{1}{k_{\perp} \rho \cos \phi} \sum_{n=-\infty}^{\infty} n B_{2 n} H_{n}^{(2)}\left(k_{\perp} \rho\right) e^{j n \varphi} \\
& E_{\varphi}^{s c}=j Z_{1} \frac{1}{\cos \phi} \sum_{n=-\infty}^{\infty} B_{2 n} H_{n}^{(2)^{\prime}}\left(k_{\perp} \rho\right) e^{j n \varphi} \\
& H_{\rho}^{s c}=-j \tan \phi \sum_{n=-\infty}^{\infty} B_{2 n} H_{n}^{(2)^{\bullet}}\left(k_{\perp} \rho\right) e^{j n \varphi} \\
& H_{\varphi}^{s c}=\frac{\tan \phi}{k_{\perp} \rho} \sum_{n=-\infty}^{\infty} n B_{2 n} H_{n}^{(2)}\left(k_{\perp} \rho\right) e^{j n \varphi} \\
& H_{z}^{s c}=\sum_{n=-\infty}^{\infty} B_{2 n} H_{n}^{(2)}\left(k_{\perp} \rho\right) e^{j n \varphi} .
\end{aligned}
$$

In the relations (20) and (21), $B_{1 n}$ and $B_{2 n}$ are amplitude coefficients of the scattered wave, which have to be determined by the boundary conditions. The total field in the external region consists of the incident plane wave (8) plus the scattered wave (20) and (21).

\subsection{The Electric Field Vector of the Incident Plane Wave Is Perpendicular to the Electron Beam}

In the case where the electric field vector of the incident plane wave is polarized perpendicularly to the axis of the beam, the particular field components of the incident plane wave that we are interested in to satisfy our boundary conditions become

$$
\begin{aligned}
& E_{\varphi}^{\mathrm{inc}}=-E^{\mathrm{inc}} \cos \varphi=-A \sum_{n=-\infty}^{\infty} j^{n-1} J_{n}^{\prime}\left(k_{\perp} \rho\right) e^{j n \varphi} \\
& H_{z}^{\mathrm{inc}}=H^{\mathrm{inc}} \cos \phi=A \frac{1}{Z_{1}} \cos \phi \sum_{n=-\infty}^{\infty} j^{n} J_{n}\left(k_{\perp} \rho\right) e^{j n \varphi} \\
& H_{\rho}^{\mathrm{inc}}=-H^{\mathrm{inc}} \sin \phi \sin \varphi=A \frac{1}{Z_{1}} \sin \phi \frac{1}{k_{\perp} \rho} \sum_{n=-\infty}^{\infty} n j^{n} J_{n}\left(k_{\perp} \rho\right) e^{j n \varphi} .
\end{aligned}
$$

For the axial components of the internal electromagnetic field and the polarization that we here consider we introduce the amplitude coefficients $\alpha_{1 n}$ and $\beta_{1 n}$ which then substitute $A_{1 n}$ and $B_{1 n}$ in relations (17). Accordingly we have

$$
\begin{aligned}
& E_{z}^{\mathrm{int}}=\sum_{n=-\infty}^{\infty} \alpha_{1 n} J_{n}\left(h_{1} \rho\right) e^{j n \varphi}+j U \Delta^{-2} Z_{0} \sum_{n=-\infty}^{\infty} \beta_{1 n} J_{n}\left(h_{2} \rho\right) e^{j n \varphi}, \\
& H_{z}^{\mathrm{int}}=j U \frac{1}{Z_{0}} \sum_{n=-\infty}^{\infty} \alpha_{1 n} J_{n}\left(h_{1} \rho\right) e^{j n \varphi}+\sum_{n=-\infty}^{\infty} \beta_{1 n} J_{n}\left(h_{2} \rho\right) e^{j n \varphi} .
\end{aligned}
$$

Correspondingly we introduce $\alpha_{2 n}$ and $\beta_{2 n}$ as the amplitude coefficients of the scattered wave. The field components of the scattered wave we obtain, without unnecessary rewriting, from relations (20) and (21) by direct substitution of $\alpha_{2 n}$ and $\beta_{2 n}$ for $A_{2 n}$ and $B_{2 n}$ respectively.

As a final remark to this section on the wave solutions of the problem we would like to point out the following. The propagation of the different modes of the field as described by the factor $e^{j\left(\omega t^{-} k_{\|}+n \varphi\right)}$, where $n$ takes positive as well as negative values in the summation, may be thought of as helical or screw waves for which the velocity of azimuthal rotation is $\frac{\omega}{n}$ and the axial phase velocity is $\frac{\omega}{k_{\|}}$. The total internal field for example is then obtained by superposition of such modes properly weighted as indicated by our formulae above. 


\section{The Amplitude Coefficients}

As boundary conditions on the surface of the cylinder of radius $a$ we take the tangential components of the electric and magnetic field vectors to be continuous through the surface.

3.1. The Magnetic Field Vector of the Incident Plane Wave Is Perpendicular to the Electron Beam

Let us introduce the following convenient notations

$$
\begin{aligned}
& d_{1}=\frac{Z_{1}}{Z_{0}} \frac{1}{q^{2} a_{2}^{2}}\left(k_{0} R+k_{\|} Q U\right) k_{0}^{2} h_{1} \cos \phi J_{n}^{\prime}\left(h_{1} a\right) H_{n}^{(2)}\left(k_{\perp} a\right)-J_{n}\left(h_{1} a\right) H_{n}^{(2) \prime}\left(k_{\perp} a\right)+\frac{Z_{1}}{Z_{0}}\left[\frac{\tan \phi}{k_{1}} U\right. \\
& \left.-\frac{1}{q^{2} a_{2}^{2}}\left(\Lambda+q^{2} U\right) k_{\|} \cos \phi\right] \frac{n}{a} J_{n}\left(h_{1} a\right) H_{n}^{(2)}\left(k_{\perp} a\right), \\
& d_{2}=\left[\frac{\tan \phi}{k_{1}}-\frac{1}{q^{2} a_{2}^{2}}\left(\Gamma+k_{0}^{3} Q U\right) \cos \phi\right] \frac{n}{a} J_{n}\left(h_{1} a\right) H_{n}^{(2)}\left(k_{\perp} a\right)-\frac{Z_{1}}{Z_{0}} U J_{n}\left(h_{1} a\right) H_{n}^{(2) \prime}\left(k_{\perp} a\right) \\
& +\frac{1}{q^{2} a_{2}^{2}}\left(\Lambda+q^{2} U\right) k_{0} h_{1} \cos \phi J_{n}^{\prime}\left(h_{1} a\right) H_{n}^{(2)}\left(k_{\perp} a\right), \\
& d_{3}=-\left[\frac{\tan \phi}{k_{1}}-\frac{1}{q^{2} a_{2}^{2}}\left(q^{2}-\Lambda \Delta^{-2} U\right) k_{\|} \cos \phi\right] \frac{n}{a} J_{n}\left(h_{2} a\right) H_{n}^{(2)}\left(k_{\perp} a\right)-\frac{Z_{0}}{Z_{1}} \Delta^{-2} U J_{n}\left(h_{2} a\right) H_{n}^{(2) '}\left(k_{\perp} a\right) \\
& -\frac{1}{q^{2} a_{2}^{2}}\left(k_{\|} Q-k_{0} R \Delta^{-2} U\right) k_{0}^{2} h_{2} \cos \phi J_{n}^{\prime}\left(h_{2} a\right) H_{n}^{(2)}\left(k_{\perp} a\right), \\
& d_{4}=J_{n}\left(h_{2} a\right) H_{n}^{(2) \prime}\left(k_{\perp} a\right)-\frac{Z_{0}}{Z_{1}} \frac{1}{q^{2} a_{2}^{2}}\left(q^{2}-\Lambda \Delta^{-2} U\right) k_{0} h_{2} \cos \phi J_{n}^{\prime}\left(h_{2} a\right) H_{n}^{(2)}\left(k_{\perp} a\right)+\frac{Z_{0}}{Z_{1}}\left[\frac{\tan \phi}{k_{1}} \Delta^{-2} U\right. \\
& \left.+\frac{1}{q^{2} a_{2}^{2}}\left(k_{0}^{3} Q-\Gamma \Delta^{-2} U\right) \cos \phi\right] \frac{n}{a} J_{n}\left(h_{2} a\right) H_{n}^{(2)}\left(k_{\perp} a\right) .
\end{aligned}
$$

The amplitude coefficients $A_{1 n}$ and $B_{1 n}$ can then be simply expressed as

$$
\begin{aligned}
& A_{1 n}=A j^{n-1} \frac{2}{\pi} \frac{1}{k_{1} a} \frac{d_{4}}{\left|\begin{array}{l}
d_{1} d_{2} \\
d_{3} d_{4}
\end{array}\right|}, \\
& B_{1 n}=A j^{n} \frac{1}{Z_{1}} \frac{2}{\pi} \frac{1}{k_{1} a} \frac{d_{2}}{\left|\begin{array}{l}
d_{1} d_{2} \\
d_{3} d_{4}
\end{array}\right|} \cdot
\end{aligned}
$$

We obtain the remaining amplitude coefficients $A_{2 n}$ and $B_{2 n}$ from

$$
\begin{aligned}
A_{2 n} & =\frac{1}{H_{n}^{(2)}\left(k_{\perp} a\right)}\left[A_{1 n} J_{n}\left(h_{1} a\right)+j \Delta^{-2} U Z_{0} B_{1 n} J_{n}\left(h_{2} a\right)+A \cos \phi j^{n} J_{n}\left(k_{\perp} a\right)\right] \\
B_{2 n} & =\frac{1}{H_{n}^{(2)}\left(k_{\perp} a\right)}\left[j U \frac{1}{Z_{0}} A_{1 n} J_{n}\left(h_{1} a\right)+B_{1 n} J_{n}\left(h_{2} a\right)\right] .
\end{aligned}
$$

3.2. The Electric Field Vector of the Incident Plane Wave Is Perpendicular to the Electron Beam field

For the polarization we here consider we have the amplitude coefficients of the internal 


$$
\begin{aligned}
& \alpha_{1 n}=-A j^{n} \frac{2}{\pi} \frac{1}{k_{1} a} \frac{d_{3}}{\mid \begin{array}{l}
d_{1} d_{2} \\
d_{3} d_{4} \mid
\end{array}} \\
& \beta_{1 n}=-A j^{n+1} \frac{1}{Z_{1}} \frac{2}{\pi} \frac{1}{k_{1} a} \frac{d_{1}}{\mid \begin{array}{l}
d_{1} d_{2} \mid \\
d_{3} d_{4}
\end{array}},
\end{aligned}
$$

and of the scattered field

$$
\begin{aligned}
\alpha_{2 n} & =\frac{1}{H_{n}^{(2)}\left(k_{\perp} a\right)}\left[\alpha_{1 n} J_{n}\left(h_{1} a\right)+j \Delta^{-2} U Z_{0} \beta_{1 n} J_{n}\left(h_{2} a\right)\right], \\
\beta_{2 n} & =\frac{1}{H_{n}^{(2)}\left(k_{\perp} a\right)}\left[j U \frac{1}{Z_{0}} \alpha_{1 n} J_{n}\left(h_{1} a\right)+\beta_{1 n} J_{n}\left(h_{2} a\right)-A \frac{1}{Z_{1}} \cos \phi j^{n} J_{n}\left(k_{\perp} a\right)\right] .
\end{aligned}
$$

\section{The Field in the Center of the Beam}

Let us consider the axial components of the electric and magnetic field vectors in the center of the beam.

4.1. The Magnetic Field Vector of the Incident Plane Wave Is Perpendicular to the Electron Beam

From the relations (17) and (25) we find for $\rho=0$,

$$
\begin{aligned}
& E_{z}(\rho=0)=A_{10}+j U \Delta^{-2} Z_{0} B_{10} \\
& =-A j \frac{2}{\pi} \frac{1}{k_{1} a} \frac{1}{\mid \begin{array}{l}
d_{1} d_{2} \\
\left.d_{3} d_{4}\right|_{n=0}
\end{array}}\left(d_{4}-U \Delta^{-2} \frac{Z_{0}}{Z_{1}} d_{2}\right)_{n=0},
\end{aligned}
$$

or

$$
\frac{E_{z}(\rho=0)}{E_{z}^{\text {in }}}=j \frac{2}{\pi} \frac{1}{k_{1} a \cos \phi} \frac{1}{\mid \begin{array}{l}
d_{1} d_{2} \\
\left.d_{3} d_{4}\right|_{n=0}
\end{array}}\left(d_{4}-U \Delta^{-2} \frac{Z_{0}}{Z_{1}} d_{2}\right)_{n=0} .
$$

Furthermore

$$
\begin{aligned}
H_{z}(\rho=0) & =j U \frac{1}{Z_{0}} A_{10}+B_{10} \\
& =A \frac{1}{Z_{1}} \frac{2}{\pi} \frac{1}{k_{1} a} \frac{1}{\mid \begin{array}{l}
d_{1} d_{2} \\
\left.d_{3} d_{4}\right|_{n=0}
\end{array}}\left(d_{2}+U \frac{Z_{1}}{Z_{0}} d_{4}\right)_{n=0}
\end{aligned}
$$

or

$$
\frac{H_{z}(\rho=0)}{H_{\perp}^{\text {inc }}}=\frac{2}{\pi} \frac{1}{k_{1} a} \frac{1}{\mid \begin{array}{l}
d_{1} d_{2} \\
\left.d_{3} d_{4}\right|_{n=0}
\end{array}}\left(d_{2}+U \frac{Z_{1}}{Z_{0}} d_{4}\right)_{n=0}
$$

where $H_{\perp}^{\text {inc }}$ is the magnetic field of the incident wave in this case.

4.2. The Electric Field Vector of the Incident Plane Wave Is Perpendicular to the Electron Beam

From eqs (23) and (28) we obtain

$$
\begin{aligned}
E_{z}(\rho=0) & =\alpha_{10}+j U \Delta^{-2} Z_{0} \beta_{10} \\
& =-A \frac{2}{\pi} \frac{1}{k_{1} a} \frac{1}{\left|\begin{array}{l}
d_{1} d_{2} \\
d_{3} d_{4}
\end{array}\right|_{n=0}}\left(d_{3}-U \Delta^{-2} \frac{Z_{0}}{Z_{1}} d_{1}\right)_{n=0},
\end{aligned}
$$


or

$$
\frac{E_{z}(\rho=0)}{E_{\perp}^{\text {inc }}}=-\frac{2}{\pi} \frac{1}{k_{1} a} \frac{1}{\left|\begin{array}{l}
d_{1} d_{2} \\
d_{3} d_{4}
\end{array}\right|_{n=0}}\left(d_{3}-U \Delta^{-2} \frac{Z_{0}}{Z_{1}} d_{1}\right)_{n=0},
$$

where $E_{\perp}^{\text {inc }}$ is the electric field of the incident wave in this case.

Correspondingly

$$
\begin{aligned}
H_{2}(\rho=0) & =j U \frac{1}{Z_{0}} \alpha_{10}+\beta_{10} \\
& =-j A \frac{1}{Z_{1}} \frac{2}{\pi} \frac{1}{k_{1} a} \frac{1}{\left|\begin{array}{l}
d_{1} d_{2} \\
d_{3} d_{4}
\end{array}\right|_{n=0}}\left(d_{1}+U \frac{Z_{1}}{Z_{0}} d_{3}\right)_{n=0}
\end{aligned}
$$

or

$$
\frac{H_{z}(\rho=0)}{H_{z}^{\text {inc }}}=-j \frac{2}{\pi} \frac{1}{k_{1} a \cos \phi} \frac{1}{\left|\begin{array}{l}
d_{1} d_{2} \\
d_{3} d_{4}
\end{array}\right|_{n=0}}\left(d_{1}+U \frac{Z_{1}}{Z_{0}} d_{3}\right)_{n=0} .
$$

\section{The Scattering Cross Sections}

We define the following three scattering cross sections,

Differential cross section:

$$
\sigma(\varphi)=\frac{\frac{1}{2} \rho \operatorname{Re}\left(E_{\varphi}^{s c} H_{z}^{s c^{*}}-E_{z}^{s c} H_{\varphi}^{s c^{*}}\right)}{\frac{1}{2} \operatorname{Re}\left(E^{\operatorname{inc}} \times H^{\operatorname{inc}}\right) \cos \phi}
$$

Total cross section:

$$
\sigma_{\mathrm{tot}}=\frac{\frac{1}{2} \rho \int_{0}^{2 \pi} d \varphi \operatorname{Re}\left(E_{\varphi}^{s c} H_{z}^{s c^{*}}-E_{z}^{s c} H_{\varphi}^{s *^{*}}\right)}{\frac{1}{2} \operatorname{Re}\left(E^{\mathrm{inc}} \times H^{\mathrm{inc}^{*}}\right) \cos \phi} .
$$

Backscattering cross section:

We here have

$$
\sigma_{B}=\frac{\frac{1}{2} \rho 2 \pi \cdot \operatorname{Re}\left(E_{\varphi}^{s c} H_{z}^{s c^{*}}-E_{2}^{s c} H_{\varphi}^{s c^{*}}\right)_{\varphi=0}}{\frac{1}{2} \operatorname{Re}\left(E^{\operatorname{lnc}} \times H^{\ln c^{*}}\right) \cos \phi} .
$$

$$
\frac{1}{2} \operatorname{Re}\left(E^{\mathrm{inc}} \times H^{\mathrm{inc}}\right) \cos \phi=A^{2} \frac{1}{Z_{1}} \cos \phi .
$$

Let us furthermore introduce the expressions for the field components of the scattered wave in the relations (34) to (36) and do the integration in (35). We then obtain the following results for the polarization where the magnetic field vector of the incident plane wave is perpendicular to the electron beam,

$$
\begin{gathered}
\sigma(\varphi)=\frac{1}{A^{2} \cos ^{2} \phi} \cdot \frac{2}{\pi k_{\perp}}\left[\left|\sum_{n=-\infty}^{\infty} A_{2 n} e^{j n}\left(\varphi+\frac{\pi}{2}\right)\right|^{2}+Z_{1}^{2}\left|\sum_{n=-\infty}^{\infty} B_{2 n} e^{j n}\left(\varphi+\frac{\pi}{2}\right)\right|^{2}\right], \\
\sigma_{\mathrm{tot}}=\frac{1}{A^{2} \cos ^{2} \phi} \cdot \frac{4}{k_{\perp}}\left[\sum_{n=-\infty}^{\infty}\left|A_{2 n}\right|^{2}+Z_{1}^{2} \sum_{n=-\infty}^{\infty}\left|B_{2 n}\right|^{2}\right] \\
\sigma_{B}=\frac{1}{A^{2} \cos ^{2} \phi} \cdot \frac{4}{k_{\perp}}\left[\left|\sum_{n=-\infty}^{\infty} j^{n} A_{2 n}\right|^{2}+Z_{1}^{2}\left|\sum_{n=-\infty}^{\infty} j B_{2 n}\right|^{2}\right] .
\end{gathered}
$$

The total scattering cross section $\sigma_{\text {tot }}=\int_{0}^{2 \pi} \sigma(\phi) d$ as expressed by relation (35) and

is the ratio between the total power scattered per unit length by the cylinder to the component of the incident power per unit area that is normal to the axis of the beam.

The backscattering cross section defined by relation (36) and explicitly expressed in the amplitude coefficients of the scattered wave by (39) we may think of as the cross section of a fictitious scatterer that scatters energy isotropically with the intensity observed as backscat- 
tering (in the plane defined by the wave-normal of the incident wave and the cylinder axis, $\varphi=0)$.

From relation (37) we notice that

$$
\sigma\left(\varphi=-\frac{\pi}{2}\right)=\frac{1}{A^{2} \cos ^{2} \phi} \frac{2}{\pi k_{\perp}}\left[\left|\sum_{n=-\infty}^{\infty} A_{2 n}\right|^{2}+Z_{1}^{2}\left|\sum_{n=-\infty}^{\infty} B_{2 n}\right|^{2}\right]
$$

To facilitate comparison between the scattering at right angle from the plane of incidence and the backscattering let us furthermore introduce

$$
\sigma_{\frac{\pi}{2}}=2 \pi \sigma\left(\varphi=-\frac{\pi}{2}\right)
$$

where $\sigma_{\frac{\pi}{2}}$ corresponds to the cross section of a fictitious scatterer that scatters isotropically with the intensity observed at right angle $\left(\varphi=-\frac{\pi}{2}\right)$ from the plane of incidence $(\varphi=0)$.

For the case where the electric field vector of the incident plane wave is perpendicular to the electron beam we obtain the results for the different cross sections from the formulae (37) to (41) by substituting $\alpha_{2 n}$ and $\beta_{2 n}$ for $A_{2 n}$ and $B_{2 n}$, respectively.

\section{Scattering of an Obliquely Incident Plane Wave by a Cold Cylindrical Plasma in a Magnetic Field}

We obtain the results in this case by making the following specifications in the relations (3),

$$
\omega_{c_{1}}=0, \quad \omega_{c_{2}}=\omega_{c}, \quad \nu_{2}=\nu,
$$

where $\omega_{c}$ and $\nu$ are the critical angular frequency and collision frequency of the electron plasma.

The expressions can then be simplified to

$$
\begin{aligned}
q^{2} & =k_{0}^{2}\left[\cos ^{2} \phi-\frac{\omega_{c}^{2}}{(\omega-j \nu)^{2}-\omega_{H}^{2}} \cdot \frac{\omega-j \nu}{\omega}\right], \\
q^{2} a_{2}^{2} & =k_{0}^{4}\left[\cos ^{2} \phi-\frac{\omega_{c}^{2}}{\omega\left(\omega-\omega_{H}-j \nu\right)}\right] \cdot\left[\cos ^{2} \phi-\frac{\omega_{c}^{2}}{\omega\left(\omega+\omega_{H}-j \nu\right)}\right] \\
\Delta^{2} & =1-\frac{\omega_{c}^{2}}{\omega(\omega-j \nu)}, \\
D^{2} & =1-\frac{\omega_{c}^{2}}{(\omega-j \nu)^{2}-\omega_{H}^{2}} \cdot \frac{\omega-j \nu}{\omega}, \\
Q & =\frac{\omega_{H}}{\omega} \frac{\omega_{c}^{2}}{(\omega-j \nu)^{2}-\omega_{H}^{2}}, \\
T^{2} & =\frac{\omega_{H}^{2}}{\omega^{2}} \cdot\left[\frac{\omega_{c}^{2}}{(\omega-j \nu)^{2}-\omega_{H}^{2}}\right]^{2}=Q^{2}, \\
p^{2} & =q^{2} D^{2}-k_{0}^{2} T^{2}, \\
\Lambda & =k_{0}^{2} \sin \phi \frac{\omega_{H}}{\omega} \cdot \frac{\omega_{c}^{2}}{(\omega-j \nu)^{2}-\omega_{H}^{2}}=k_{0}^{2} \sin \phi \cdot Q \\
R & =\frac{p^{2}}{k_{0}^{2}} \\
\Gamma & =k_{0} q^{2} \sin \phi .
\end{aligned}
$$


For $p^{2}-q^{2} \Delta^{2}$ that enters $h_{1}, h_{2}$, and $U$ we have in this case the simple result

and accordingly

$$
p^{2}-q^{2} \Delta^{2}=-k_{\perp}^{2} \frac{\omega_{H}}{\omega-j \nu} \cdot Q
$$

$$
\begin{aligned}
& U=\frac{1}{2 \sin \phi}\left[\frac{\omega_{H}}{\omega-j \nu} \cos ^{2} \phi-\sqrt{\frac{\omega_{H}^{2}}{(\omega-j \nu)^{2}} \cos ^{4} \phi+4 \Delta^{2} \sin ^{2} \phi}\right], \\
& h_{1}^{2}=\frac{1}{2 D^{2}}\left[p^{2}+q^{2} \Delta^{2}+Q k_{0}^{2} \sqrt{\left.\frac{\omega_{H}^{2}}{(\omega-j \nu)^{2}} \cos ^{4} \phi+4 \Delta^{2} \sin ^{2} \phi\right]}\right. \\
& h_{2}^{2}=\frac{1}{2 D^{2}}\left[p^{2}+q^{2} \Delta^{2}-Q k_{0}^{2} \sqrt{\left.\frac{\omega_{H}^{2}}{(\omega-j \nu)^{2}} \cos ^{4} \phi+4 \Delta^{2} \sin ^{2} \phi\right]}\right.
\end{aligned}
$$

\section{Results of Machine Computation}

Extensive machine computations have been made for the case of the cylindrical electron plasma neglecting the effect of collisions.

In order to demonstrate the possibility of utilizing the results of this investigation for diagnostic purposes let us consider here only the results for a particular case.

For the polarization where the magnetic field vector of the incident plane wave is perpendicular to the axis of the cylindrical plasma we study the backscattering cross section as a function of the angle of incidence of the plane wave. In figures 2 and 3 we have taken $\omega_{p}>$ $\omega_{H}>\omega$, where $\omega_{p}$ is the plasma frequency $\left(\omega_{p}=\omega_{c}\right)$.

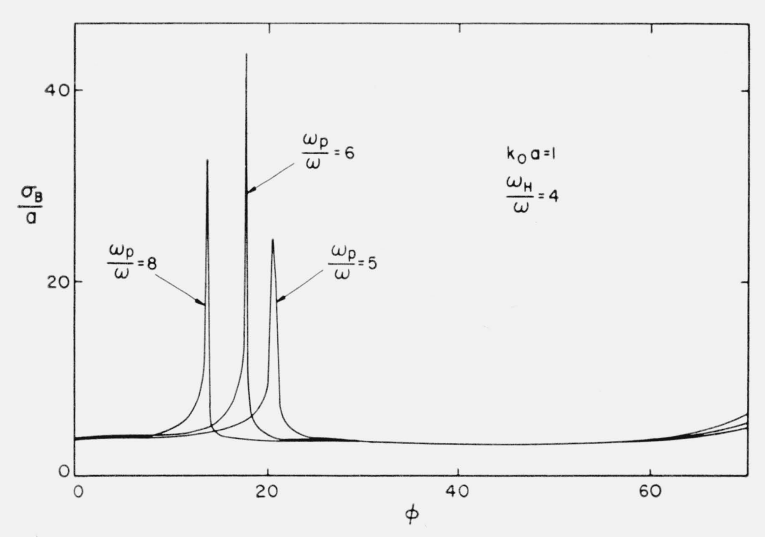

FIGURE 2. The backscattering cross section over the radius of the plasma cylinder as a function of the angle of incidence of the plane wave for different values of $\frac{\omega_{\mathrm{p}}}{\omega}$.

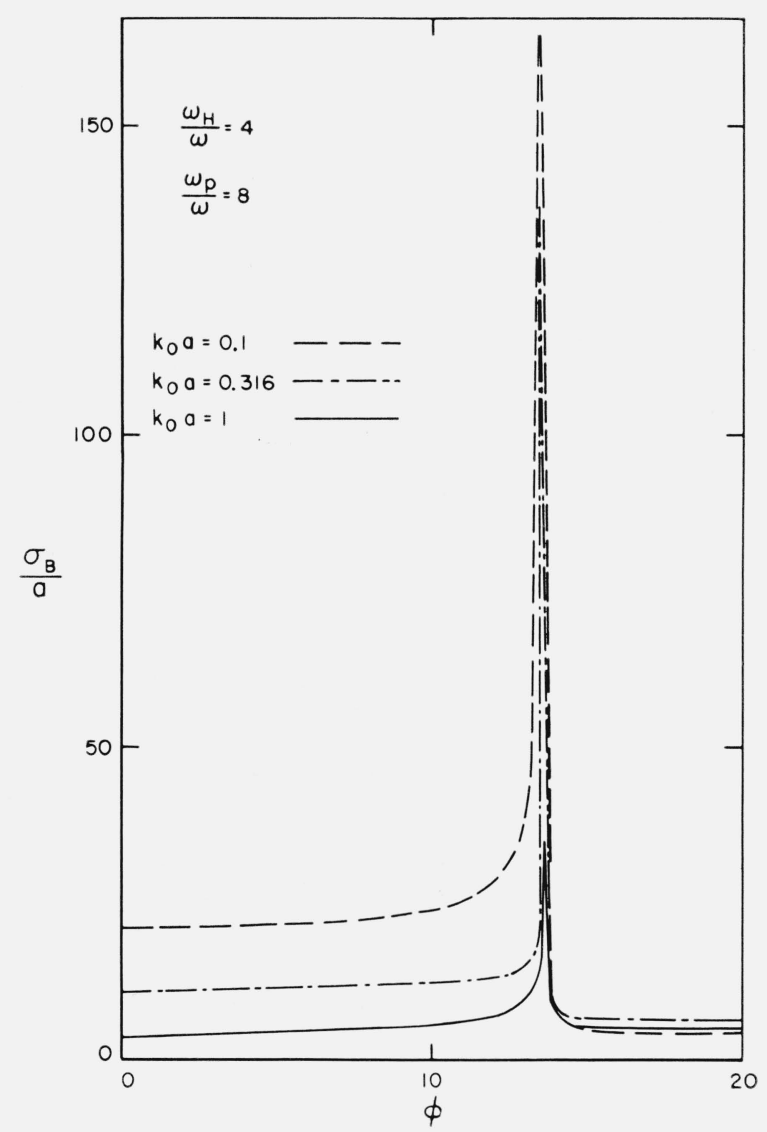

FiguRE 3. The backscattering cross section over the radius of the plasma cylinder as a function of the angle of incidence of the plane wave for different values of $\mathrm{k}_{0} \mathrm{a}$. 
Figure 2 clearly demonstrates that resonances occur in the backscattering cross section at different angles of incidence for different values of the plasma frequency, i.e., the density of the plasma cylinder, and a fixed value of the gyrofrequency, i.e., the magnetic field. In figure 2 we have taken $\frac{\omega_{p}}{\omega}$ equal to three different values 8,6 , and 5 for $\frac{\omega_{H}}{\omega}=4$ and $k_{0} a=1$. Measurements of the values of the resonance angles might possibly be useful to determine the density of the plasma cylinder.

In figure 3 we have plotted backscattering curves for three different values of $k_{0} a$ and fixed values of $\frac{\omega_{p}}{\omega}$ and $\frac{\omega_{H}}{\omega}$. Apparently there is no observable change in the value of the resonance angle when $k_{0} a$ varies between 0.1 and 1 .

It remains to be shown by experiment that resonances of the kind predicted by these calculations do play a role for a cylindrical cold plasma and if so whether or not they can be utilized for diagnostics of such a plasma.

The machine computations were made on the IBM 704 computer in New York, on time available for the Plasma Physics Laboratory of Princeton University, of which the author was a member during the year 1960-61. It is a pleasure to acknowledge the very generous help of L. Hoffman, M. Sc., who did all the coding work. The work was finally written up at Research Laboratory of Electronics, Chalmers University of Technology, Gothenburg, Sweden. It forms a continuation of earlier work by the author as a part of the general electronic research program of the Research Laboratory of Electronics.

\section{References}

Wilhelmsson, H., The interaction between an obliquely incident plane electromagnetic wave and an electron beam, Part I, Trans. Chalmers Univ. of Tech., No. 155 (Report No. 32, Research Lab. of Electronics) (1954).

Wilhelmsson, H., The interaction between an obliquely incident plane electromagnetic wave and an electron beam, Part II, Trans. Chalmers Univ. of Tech., No. 198 (Report No. 45, Research Lab. of Electronics) (1958a).

Wilhelmsson, H., The interaction between an obliquely incident plane electromagnetic wave and an electron beam, Part III, Trans. Chalmers Univ. of Tech, No. 206 (Report No. 47, Research Lab. of Electronics) (1958b).

Wilhelmsson, H., On the properties of the electron beam in the presence of an axial magnetic field of arbitrary strength, Trans. Chalmers Univ. of Tech., No. 205 (Report No. 46, Research Lab. of Electronics) (1958c).

\section{Additional References}

Platzman, P. M., and Ozaki, H. T., Scattering of electromagnetic waves from an infinitely long magnetized cylindrical plasma, J. Appl. Phys., 31, No. 9 (Sept. 1960).

Wait, J. R., Some boundary value problems involving plasma media, J. Research NBS 65B (Math. and Math. Phys.) No. 2 (Apr.-June 1961).

Sirkis, M. D., Strain, R. J., and Kunz, W. E., Electron beam excitation of a Fabry-Perot interferometer, J. Appl. Phys. 32, No. 10, 2055-2056 (Oct. 1961).

(Paper 66D4-206) 